\title{
Polyvascular Disease in Patients Presenting with Acute Coronary Syndrome: Its Predictors and Outcomes
}

\author{
Hassan Al Thani, ${ }^{1,2}$ Ayman El-Menyar, ${ }^{1,2}$ Khalid F. AlHabib, ${ }^{3}$ \\ Ahmed Al-Motarreb, ${ }^{4}$ Ahmad Hersi, ${ }^{3}$ Hussam AlFaleh, ${ }^{3}$ Nidal Asaad, ${ }^{1,2}$ Shukri Al Saif, ${ }^{5}$ \\ Wael Almahmeed, ${ }^{6}$ Kadhim Sulaiman, ${ }^{7}$ Haitham Amin, ${ }^{8}$ Alawi A. Alsheikh-Ali, ${ }^{6,9}$ \\ Khalid AlNemer, ${ }^{10}$ and Jassim Al Suwaidi ${ }^{1,2}$ \\ ${ }^{1}$ Department of Cardiology and Cardiovascular Surgery, Hamad General Hospital, Doha 3050, Qatar \\ ${ }^{2}$ Department of Clinical Medicine, Weill Cornell Medical College, Doha 24144, Qatar \\ ${ }^{3}$ Department of Cardiology, King Fahad Cardiac Centre, College of Medicine, King Saud University, Riyadh 11472, Saudi Arabia \\ ${ }^{4}$ Faculty of Medicine, Sana'a University, Sana'a, Yemen \\ ${ }^{5}$ Department of Cardiology, Saud AllBabtain Cardiac Centre, Dammam 11850, Saudi Arabia \\ ${ }^{6}$ Institute of Cardiac Sciences, Sheikh Khalifa Medical City, Abu Dhabi, UAE \\ ${ }^{7}$ Department of Cardiology, Royal Hospital, Muscat, Oman \\ ${ }^{8}$ Mohammed Bin Khalifa Cardiac Centre, Bahrain \\ 9 Tufts Clinical and Translational Science Institute, Tufts University School of Medicine, Boston, MA 02111, USA \\ ${ }^{10}$ Department of Cardiology, Security Forces Hospital, Riyadh, Saudi Arabia
}

Correspondence should be addressed to Hassan Al Thani, althanih@hotmail.com

Received 9 October 2011; Accepted 2 November 2011

Academic Editors: A. J. Kirtane and L. Kuller

Copyright ( $) 2012$ Hassan Al Thani et al. This is an open access article distributed under the Creative Commons Attribution License, which permits unrestricted use, distribution, and reproduction in any medium, provided the original work is properly cited.

\begin{abstract}
We evaluated prevalence and clinical outcome of polyvascular disease (PolyVD) in patients presenting with acute coronary syndrome (ACS). Data for 7689 consecutive ACS patients were collected from the 2nd Gulf Registry of Acute Coronary Events between October 2008 and June 2009. Patients were divided into 2 groups (ACS with versus without PolyVD). All-cause mortality was assessed at 1 and 12 months. Patients with PolyVD were older and more likely to have cardiovascular risk factors. On presentation, those patients were more likely to have atypical angina, high resting heart rate, high Killip class, and GRACE risk scoring. They were less likely to receive evidence-based therapies. Diabetes mellitus, renal failure, and hypertension were independent predictors for presence of PolyVD. PolyVD was associated with worse in-hospital outcomes (except for major bleedings) and all-cause mortality even after adjusting for baseline covariates. Great efforts should be directed toward primary and secondary preventive measures.
\end{abstract}

\section{Introduction}

Atherothrombosis is a systemic disease that often occurs at more than one vascular site and should be considered in practice as an integral disease [1-3]. Moreover, patients with affected arterial disease are more likely to develop higher event rates than patients with multiple risk factors only $[3,4]$. Polyvascular disease (PolyVD) defined as presence of more than one affected vascular bed, that is, any combination of the following: coronary artery disease (CAD), peripheral arterial disease (PAD), and cerebrovascular disease (CVD) [5-9]. The frequency and impact of PolyVD in patients with acute coronary syndrome (ACS) in the Gulf region of the Middle East have not been studied yet. These countries have higher prevalence of the traditional risk factors in a unique fashion [1]. The aim of the current study is to evaluate the 
prevalence of PolyVD and its impact on the in-hospital major adverse events and 1-year mortality across ACS in a Middle Eastern population.

\section{Methods}

2.1. Study Population. Data were collected from a prospective, multicenter study of the 2nd Gulf Registry of Acute Coronary Events (Gulf RACE-2) between October 2008 and June 2009. We recruited 7,930 consecutive patients with ACS from 6 adjacent Middle Eastern Gulf countries (Bahrain, Kingdom of Saudi Arabia, Qatar, Oman, United Arab Emirates, and Yemen). Patients diagnosed with ACS, including unstable angina (UA) and non-ST- and ST-elevation myocardial infarction (NSTEMI and STEMI, resp.), were enrolled from 65 hospitals. On-site cardiac catheterization laboratory was available in $43 \%$ of the participating hospitals. All prospective patients with ACS were eligible for enrollment. The study received ethics approval from the institutional review boards in all participating hospitals. Full details of the methods have been previously published [10].

2.2. Definitions. ACS: diagnosis of the different types of ACS and definitions of data variables were based on the American College of Cardiology clinical data standards [10, 11]. Peripheral arterial disease (PAD): defined as presence of any of the following: intermittent claudication, critical limb ischemia (ulcer or gangrene), peripheral bypass surgery (surgical bypass for PAD indication), or peripheral percutaneous transluminal angioplasty. Polyvascular disease (PolyVD) was defined as presence of more than one affected vascular bed, that is, CAD, cerebrovascular disease (CVD), and asymptomatic or symptomatic peripheral arteries (PAD) [6]. A case report form (CRF) for each patient with suspected ACS was filled out upon hospital admission by assigned physicians and/or research assistants using standard definitions and was completed throughout the patient's hospital stay. All CRFs were verified by a cardiologist then sent online to the principal coordinating center, where the forms were further checked for mistakes before submission for final analysis.

2.3. Statistical Analysis. Data are presented as proportions or mean \pm standard deviation (SD) as appropriate. Baseline demographic characteristics, past medical history, clinical presentation, and clinical outcomes were compared between 2 groups (ACS with versus without PolyVD). Subanalysis was performed comparing the clinical outcomes among various combinations of vascular bed affection (ACS alone, ACS plus PAD, ACS plus CVD, and ACS plus PAD and CVD). Statistical analyses were conducted using the Student's $t$-test for continuous variables and Pearson chi-square $\left(\chi^{2}\right)$ test for categorical variables. Primary endpoints included recurrent ischemia, heart failure (HF), stroke, major bleedings, and mortality. All-cause mortality was assessed at 1-month and 12-month follow-up period. In order to assess the independent association of PolyVD with clinical outcomes, logistic regression analysis models were used and crude and adjusted odd ratios were calculated. We included significant baseline variables in the analysis such as age, sex, diabetes mellitus, hypertension, dyslipidemia, smoking, renal failure, prior coronary artery disease and prior coronary revascularization in addition to the type of ACS at presentation. Univariate and multivariate analysis for clinical outcomes were tested. Global Registry of ACS events (GRACE) risk scores for hospital mortality were used to stratify the risk status of patients at presentation as low, intermediate, or high [12]. All $P$ values were the results of 2 -tailed tests and values $<0.05$ were considered significant. Data analysis was carried out using the Statistical Package for Social Sciences version 18 (SPSS Inc., USA).

\section{Results}

3.1. Clinical and Biochemical Profiles. Out of the 7689 patients who were admitted with ACS, PolyVD was documented in 428 patients $(5.6 \%)$ in terms of ACS plus PAD (110 patients), ACS plus CVD (284 patients), and ACS plus PAD and CVD (34 patients). Table 1 shows the baseline characteristics and risk factors of patients with PolyVD including ACS in comparison to ACS-alone patients. Patients with PolyVD were 6 years older $(62 \pm 11$ versus $56 \pm 12$, $P<0.0001)$ and were more likely to be female (31\% versus $20 \%, P=0.001)$. PolyVD patients were more likely to have risk factors such as diabetes mellitus (67\% versus $38 \%$, $P=0.001)$, hypertension (78\% versus $45 \%, P=0.001)$, dyslipidemia (55\% versus $36 \%, P=0.001$ ), and renal failure (14\% versus $3 \%, P=0.001$ ). They were less likely to be smokers ( $45 \%$ versus $54 \%, P=0.001)$. On presentation with ACS, PolyVD patients had higher heart rate, and GRACE risk score and were more likely to present with a higher Killip class $(P=0.001$ for all). NSTEACS was the most frequent diagnosis in PolyVD patients whereas STEMI was the predominant diagnosis in ACS-alone group.

3.2. In-Hospital Treatment Pattern. Table 2 demonstrates the treatment pattern for patients with and without PolyVD. In regard to on-admission therapy, there were no differences between the two groups in the use of heparin and glycoprotein inhibitors. Apart from thrombolysis therapy use that was in favor of ACS-alone group, patients with PolyVD were more likely to be treated with oral antiplatelet, angiotensin-converting enzyme inhibitors, statin, and $\beta$ blockers. Coronary intervention was more frequently used in ACS-alone group in comparison to PolyVD patients. Aspirin, statin, and $\beta$-blockers were more frequently used during hospitalization and at discharge in ACS-alone patients.

3.3. Predictors for Polyvascular Disease. Multivariate logistic regression analysis showed that the important independent predictors for the presence of PolyVD in ACS patients were diabetes mellitus (adjusted OR 2.28; 95\% CI 1.81-2.89), renal failure (adjusted OR 2.32; 95\% CI 1.54-3.03) and hypertension (adjusted OR 2.66; 2.03-3.49), $P=0.001$ for all (Table 3).

3.4. Clinical Outcomes. Figure 1 demonstrates the clinical outcomes in different vascular disease combinations. Table 2 shows hospital outcomes in PolyVD versus ACS-alone group. All primary endpoints were significantly worse in PolyVD 
TABLE 1: Risk factors and clinical and laboratory findings in patients with acute coronary syndrome with and without polyvascular disease.

\begin{tabular}{|c|c|c|c|}
\hline & $\begin{array}{l}\text { ACS alone } \\
(n=7261)\end{array}$ & $\begin{array}{c}\text { Polyvascular disease } \\
(n=428)\end{array}$ & $\begin{array}{c}P \\
\text { value }\end{array}$ \\
\hline Age & $56 \pm 12$ & $62 \pm 12$ & 0.001 \\
\hline Female & 20 & 31 & 0.001 \\
\hline Diabetes mellitus & 38 & 67 & 0.001 \\
\hline Hypertension & 45.3 & 78 & 0.001 \\
\hline Dyslipidemia & 36 & 55 & 0.001 \\
\hline Smoking & 54 & 45 & 0.001 \\
\hline Renal failure & 3 & 14 & 0.001 \\
\hline $\begin{array}{l}\text { Prior coronary artery } \\
\text { disease }\end{array}$ & 39 & 64 & 0.001 \\
\hline Prior revascularization & 11 & 25 & 0.001 \\
\hline Khat chewing & 19 & 20 & 0.92 \\
\hline \multicolumn{4}{|l|}{ Clinical presentation } \\
\hline $\begin{array}{l}\text { Atypical presentation } \\
\text { ACS }\end{array}$ & 15 & 28 & 0.001 \\
\hline Heart rate & $84 \pm 20$ & $90 \pm 22$ & 0.001 \\
\hline Systolic blood pressure & $135 \pm 29$ & $140 \pm 35$ & 0.01 \\
\hline $\begin{array}{l}\text { Diastolic blood } \\
\text { pressure }\end{array}$ & $81 \pm 17$ & $79 \pm 20$ & 0.02 \\
\hline Unstable angina & 24 & 24 & \\
\hline Non-STEMI & 29 & 45 & 0.001 \\
\hline STEMI & 47 & 31 & \\
\hline Killip class $>1$ & 21 & 45 & 0.001 \\
\hline Low GRACE risk score & 41 & 19 & \\
\hline High GRACE risk score & 20 & 43 & 0.001 \\
\hline \multicolumn{4}{|l|}{ Laboratory findings } \\
\hline $\begin{array}{l}\text { Fasting blood sugar } \\
(\mathrm{mmol} / \mathrm{L})\end{array}$ & $7.3 \pm 3$ & $8.1 \pm 3$ & 0.001 \\
\hline $\begin{array}{l}\text { First hemoglobin } \\
(\mathrm{g} / \mathrm{dL})\end{array}$ & $13.7 \pm 2$ & $12.5 \pm 2$ & 0.001 \\
\hline $\begin{array}{l}\text { Serum creatinine } \\
\text { (umol/L) }\end{array}$ & $100 \pm 72$ & $130 \pm 110$ & 0.001 \\
\hline Hemoglobin A1c (\%) & $7.6 \pm 2.4$ & $8 \pm 2.4$ & 0.15 \\
\hline $\begin{array}{l}\text { Total cholesterol } \\
(\mathrm{mmol} / \mathrm{L})\end{array}$ & $4.9 \pm 1.6$ & $4.4 \pm 1.6$ & 0.001 \\
\hline $\begin{array}{l}\text { Serum triglyceride } \\
(\mathrm{mmol} / \mathrm{L})\end{array}$ & $1.8 \pm 1.1$ & $1.6 \pm 0.8$ & 0.001 \\
\hline $\begin{array}{l}\text { Low-density } \\
\text { lipoprotein }\end{array}$ & $3.2 \pm 1.3$ & $2.8 \pm 1.2$ & 0.001 \\
\hline $\begin{array}{l}\text { High-density } \\
\text { lipoprotein }\end{array}$ & $1.05 \pm 0.5$ & $0.99 \pm 0.4$ & 0.01 \\
\hline Peak troponin $\mathrm{T}$ & $1.4 \pm 0.7$ & $1.3 \pm 0.6$ & 0.02 \\
\hline $\begin{array}{l}\text { LV ejection fraction } \\
<50 \%\end{array}$ & 73 & 85 & 0.001 \\
\hline 1-vessel CAD & 29 & 48 & 0.001 \\
\hline 2-vessel CAD & 25 & 18 & 0.12 \\
\hline 3-vessel CAD & 30 & 17 & 0.004 \\
\hline
\end{tabular}

All categorical and continuous variables are given in percent and mean \pm $\mathrm{SD}$, respectively.

except for major bleedings. One- and 12-month all-cause mortality was 2-times greater in PolyVD group. Univariate analysis showed that PolyVD was predictor for reischemia,
TABLE 2: Management and clinical outcomes in patients with acute coronary syndrome with and without polyvascular disease.

\begin{tabular}{|c|c|c|c|}
\hline & $\begin{array}{l}\text { ACS alone } \\
(n=7261)\end{array}$ & $\begin{array}{c}\text { Polyvascular } \\
\text { disease } \\
(n=428)\end{array}$ & $P$ value \\
\hline \multicolumn{4}{|l|}{ On admission therapy $\%$} \\
\hline Aspirin & 38 & 74 & 0.001 \\
\hline Clopidogrel & 11 & 23 & 0.001 \\
\hline$\beta$-blockers & 28 & 43.5 & 0.001 \\
\hline ACE inhibitors & 24 & 46 & 0.001 \\
\hline Statins & 29 & 58 & 0.001 \\
\hline Thrombolysis* & 52 & 20 & 0.001 \\
\hline Unfractionated heparin & 42 & 40 & 0.46 \\
\hline LMW heparin & 37 & 41 & 0.12 \\
\hline Glycoprotein inhibitors & 7.7 & 8 & 0.82 \\
\hline \multicolumn{4}{|l|}{ During hospitalization $\%$} \\
\hline Aspirin & 98.5 & 96 & 0.001 \\
\hline Clopidogrel & 76 & 81 & 0.02 \\
\hline$\beta$ blockers & 75 & 68 & 0.002 \\
\hline ACE inhibitors & 71 & 69 & 0.31 \\
\hline Statins & 95 & 92.5 & 0.03 \\
\hline Coronary angiography & 39 & 31 & 0.002 \\
\hline PCI & 22 & 12.4 & 0.001 \\
\hline \multicolumn{4}{|l|}{ Discharge medications $\%$} \\
\hline Aspirin & 96 & 92 & 0.001 \\
\hline Clopidogrel & 68 & 70 & 0.32 \\
\hline$\beta$ blockers & 80 & 74 & 0.01 \\
\hline ACE inhibitors & 72 & 63.5 & 0.001 \\
\hline Statins & 92 & 85 & 0.001 \\
\hline \multicolumn{4}{|l|}{ Outcomes \% } \\
\hline Reischemia & 15 & 23.4 & 0.001 \\
\hline Heart failure & 11.6 & 29.7 & 0.001 \\
\hline Stroke & 0.6 & 2.3 & 0.001 \\
\hline Bleedings & 0.6 & 0.9 & 0.31 \\
\hline Hospital death & 4.2 & 9.1 & 0.001 \\
\hline 1-month death & 7.4 & 16.3 & 0.001 \\
\hline 12-month death & 11.2 & 24.6 & 0.001 \\
\hline
\end{tabular}

${ }^{*}$ ST-elevation MI, PCI: percutaneous coronary intervention.

HF, stroke, and hospital, 1 , and 12-month mortality $(P=$ 0.001 for all). Similarly, multivariate logistic regression analysis denoted that PolyVD was an independent predictor for all outcomes except for major bleeding. PolyVD was strong predictor for in-hospital stroke (adjusted OR 5.40; $95 \%$ CI 2.17-13.29, $P=0.001$ Table 4 and Figure 2).

3.5. Gender and Polyvascular Disease. In comparison to their counterparts with ACS alone, men and women with PolyVD were older and had higher percent of commodities except for smoking. When compared to men, women with ACS alone had significant worse outcomes in terms of re-ischemia ( $18 \%$ versus $14 \%)$, in-hospital mortality (6.3\% versus $3.7 \%)$, 1 month mortality $(9.7 \%$ versus $6.8 \%), P=0.001$ for all. In 
TABLE 3: Multivariate logistic regression analysis for clinical predictors of polyvascular disease in patients presenting with acute coronary syndrome.

\begin{tabular}{lcc}
\hline & Odds ratio (95\% confidence interval) & $P$ value \\
\hline Age & $1.04(1.036-1.056)$ & 0.001 \\
Male gender & $1.13(0.865-1.466)$ & 0.34 \\
Dyslipidemia & $1.10(0.882-1.392)$ & 0.37 \\
Diabetes & $2.28(1.809-2.889)$ & 0.001 \\
mellitus & $2.66(2.028-3.489)$ & 0.001 \\
Hypertension & $1.24(0.970-1.591)$ & 0.07 \\
Smoking & $1.003(0.986-1.020)$ & 0.62 \\
Obesity & $2.32(1.543-3.029)$ & 0.001 \\
Renal failure &
\end{tabular}

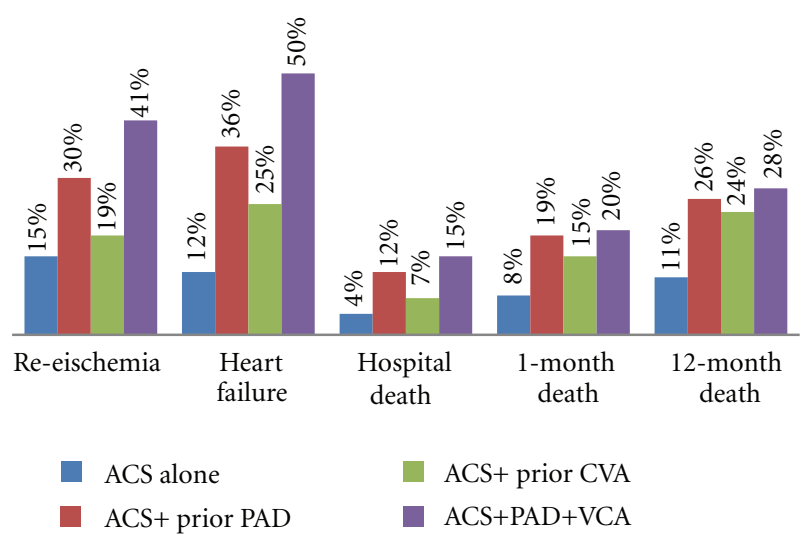

FIGURE 1: Clinical outcomes in different vascular disease combinations ( $P=0.001$ for all comparisons).

all forms of PolyVD, there were no significant morbidity and mortality differences between men and women (Table 5).

Table 6 shows risk factors and in-hospital mortality in patients with versus without polyvascular disease presenting with acute coronary syndrome in 3 major clinical studies.

\section{Discussion}

The present study reports on the frequency, predictors and implication of PolyVD in patients presenting with ACS in the Gulf Region of the Middle East. Up to our knowledge, PolyVD in ACS patients was not reported in the Middle East before. There are several key findings in this study. First, PolyVD is a common disorder in that region in the setting of acute coronary events and represents a marker of high-risk population. The prevalence of PolyVD in the current study $(5.6 \%)$ is over 2-fold lower versus GRACE study (15.6\%), Alliance project (13\%), and MASCARA study (16.6\% Table 6). Among the 4 studies, the high percent of young age, DM, and renal failure were observed in the current study. However, the difference in presentations and outcomes in the 4 studies may in part relate to diversity of biological and environmental factors between the different ethnicities. This observation needs further confirmatory studies. Second, PolyVD is associated with higher mortality

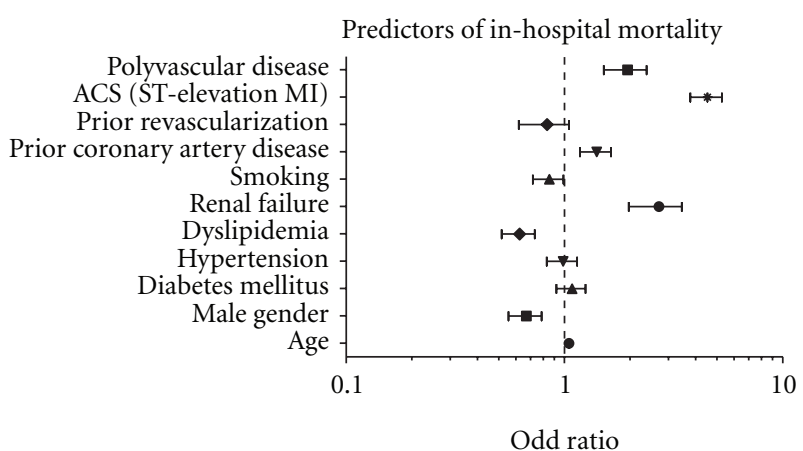

(a)

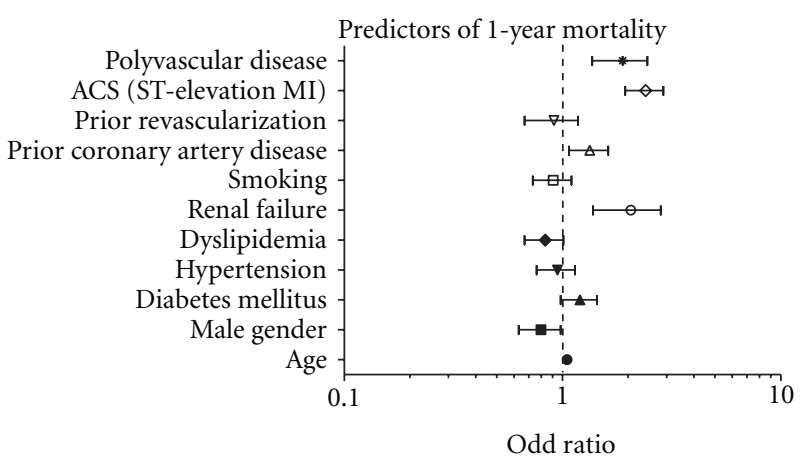

(b)

FIGURE 2: Clinical predictors for in-hospital and 1-year mortality.

rate even after adjusting for baseline variables. Third, at presentation, those patients were more likely to have atypical presentation, high resting heart rate, high Killip class, and high GRACE risk score. Moreover, NSTEACS was the most frequent diagnosis in PolyVD in comparison to ACS-alone group. Our findings are consistent with data from Western reports [5-9]. These studies showed that the management of PolyVD group was less aggressive in terms of less in-hospital coronary intervention and less evidence-based therapies during hospitalization and at discharge [6-8]. Several factors could explain the undertreatment trend in PolyVD group such as high percent of comorbidities with subsequent contraindication of some medications, physician and patient discretion, and possible socioeconomic factors. Understanding such reasons may reduce this therapy imbalance. Fourth, the current study shows that increasing the number of the affected vascular bed is associated with the worst clinical scenario (Figure 1). Apart from major bleedings, PolyVD is independent predictor for all in-hospital adverse outcomes. PolyVD increased risk of stroke 5-times even after adjustment for other covariates. PAD increased rate of $\mathrm{HF}$ and hospital mortality by 3-times whereas presence of CVD increased rate of in-hospital stroke 4-times. Data from Table 5 indicates that the highest mortality rate was observed in patients with PAD in our study, in patients with CVD in ALLIANCE project, and in patients with triple vascular bed affection in MASCARA and our study. Data from the Japanese REACH registry [13] denoted that the rates of 1-year stroke and myocardial infarction were higher 
TABLE 4: Multivariate logistic regression analysis for predictors of clinical outcomes in patients with polyvascular disease presenting with acute coronary syndrome.

\begin{tabular}{lcc}
\hline & Unadjusted odds ratio $(95 \% \mathrm{CI})$ & Adjusted odds ratio* $(95 \% \mathrm{CI})$ (95) \\
Re-ischemia & $1.7(1.37-2.15), P=0.001$ & $1.50(1.12-2.01), P=0.007$ \\
Heart failure & $3.3(2.67-4.09), P=0.001$ & $2.00(1.47-2.61), P=0.001$ \\
In-hospital Stroke & $4.1(2.03-8.22), P=0.001$ & $5.40(2.17-13.29), P=0.001$ \\
Major bleedings & $1.61(0.57-4.52), P=0.36$ & $1.68(0.54-5.17), P=0.37$ \\
Hospital death & $2.3(1.66-3.26), P=0.001$ & $1.85(1.25-2.74), P=0.01$ \\
1-month death & $2.5(1.89-3.29), P=0.001$ & $2.03(1.46-2.83), P=0.001$ \\
12-month death & $2.6(2.02-3.32), P=0.001$ & $1.83(1.83-2.45), P=0.003$ \\
\hline
\end{tabular}

CI: confidence interval, * variables adjusted for age, sex, diabetes mellitus, hypertension, dyslipidemia, smoking, renal failure, prior coronary artery disease, and prior coronary revascularization in addition to the type of ACS at presentation.

TABLE 5: Clinical profiles, admission therapy, and outcomes in men and women.

\begin{tabular}{|c|c|c|c|c|c|c|}
\hline & \multicolumn{3}{|c|}{ ACS alone } & \multicolumn{3}{|c|}{ Polyvascular disease } \\
\hline & Men & Women & $P$ value & Men & Women & $P$ value \\
\hline Age (yrs, mean) & $55 \pm 12$ & $61 \pm 12$ & 0.001 & $64 \pm 12$ & $65 \pm 13$ & 0.19 \\
\hline Diabetes mellitus \% & 35 & 51 & 0.001 & 63 & 74 & 0.03 \\
\hline Hypertension \% & 40.5 & 64 & 0.001 & 76 & 81 & 0.24 \\
\hline Dyslipidemia \% & 34 & 44 & 0.001 & 53 & 63 & 0.55 \\
\hline Smoking \% & 65 & 10 & 0.001 & 57 & 16 & 0.001 \\
\hline Renal failure $\%$ & 3 & 5 & 0.001 & 16 & 13 & 0.44 \\
\hline \multicolumn{7}{|l|}{ Admission therapy (\%) } \\
\hline Aspirin & 98.6 & 98.5 & 0.75 & 96 & 97 & 0.51 \\
\hline Clopidogrel & 79 & 64 & 0.001 & 82 & 79 & 0.51 \\
\hline Beta blockers & 76 & 71 & 0.001 & 70 & 63 & 0.11 \\
\hline ACE inhibitors & 71 & 72 & 0.72 & 70 & 65 & 0.31 \\
\hline Heparin & 58 & 59 & 0.98 & 41 & 37 & 0.50 \\
\hline Glycoprotein inhibitors & 8.3 & 5.2 & 0.001 & 9 & 6 & 0.23 \\
\hline Statin & 95 & 94 & 0.01 & 92 & 94 & 0.57 \\
\hline PCI & 23 & 19 & 0.01 & 13 & 10.5 & 0.55 \\
\hline \multicolumn{7}{|l|}{ Outcomes (\%) } \\
\hline Reischemia & 14 & 18 & 0.001 & 21 & 27 & 0.17 \\
\hline Heart failure & 10.7 & 15.5 & 0.001 & 29 & 34 & 0.28 \\
\hline Hospital mortality & 3.7 & 6.3 & 0.001 & 8.6 & 10.8 & 0.46 \\
\hline 1-month mortality & 6.8 & 9.7 & 0.001 & 15 & 20 & 0.26 \\
\hline 12-month mortality & 10.3 & 15 & 0.001 & 23 & 29 & 0.22 \\
\hline
\end{tabular}

for patients with CVD and PAD than for patients with CVD and $\mathrm{CAD}$; however, that study was not carried out during the acute setting of coronary artery disease and did not report on the in-hospital rate of stroke. Fifth, the present study expands the previous reports and shows that diabetes mellitus, hypertension, and renal failures are independent predictors for the presence of PolyVD. This finding highlights the importance of primary and secondary prevention in this high-risk population. Sixth, male gender has better outcomes in ACS alone in comparison to female. These unfavorable outcomes associated with female gender were shown in our previous work as well [14]. Interestingly, presence of PolyVD is not associated with significant differences in the outcomes between men and women, this finding warrants further exploration.

\section{Limitation}

The current study is an observational study; however, welldesigned observational studies may provide valid results. Another limitation of the current retrospective analysis is that the diagnosis of PAD relied on the clinical history and not on the measurement of ankle-brachial index (ABI). This limitation could be explained in part by facts that 
TABLE 6: Risk factors and in-hospital mortality in patients with versus without polyvascular disease presenting with acute coronary syndrome in different clinical studies.

\begin{tabular}{|c|c|c|c|c|c|c|c|c|c|c|c|c|c|c|c|c|}
\hline & \multicolumn{4}{|c|}{ GRACE $(n=32735)[7]$} & \multicolumn{4}{|c|}{ MASCARA $(n=6745)[9]$} & \multicolumn{4}{|c|}{ GULFRACE-2 $(n=7689)$} & \multicolumn{4}{|c|}{ ALLIANCE $(n=8904)[6]$} \\
\hline & \multirow{2}{*}{ A } & \multicolumn{3}{|c|}{ PolyVD (15.6\%) } & \multirow{2}{*}{ A } & \multicolumn{3}{|c|}{ PolyVD (16.6\%) } & \multirow{2}{*}{ A } & \multicolumn{3}{|c|}{ PolyVD (5.6\%) } & \multirow{2}{*}{ A } & \multicolumn{3}{|c|}{ PolyVD (13\%) } \\
\hline & & $\mathrm{B}$ & $\mathrm{C}$ & $\mathrm{D}$ & & $\mathrm{B}$ & $\mathrm{C}$ & $\mathrm{D}$ & & $\mathrm{B}$ & $\mathrm{C}$ & $\mathrm{D}$ & & $\mathrm{B}$ & $\mathrm{C}$ & $\mathrm{D}$ \\
\hline Patients \% & 84 & 7 & 6 & 2 & 83 & 9 & 6 & 2 & 94 & 1 & 4 & 0.6 & 87 & 8 & 4 & 1 \\
\hline Age (mean yrs) & 64 & 71 & 73 & 73 & 67 & 70 & 73 & 72 & 56 & 63 & 65 & 66 & 65 & & 72 & \\
\hline Smoking & 59 & 69 & 53 & 68 & $36 / 28^{*}$ & $61 / 22^{*}$ & $44 / 16^{*}$ & $60 / 21^{*}$ & 54 & 54 & 39 & 62 & 59 & & 63 & \\
\hline $\begin{array}{l}\text { Diabetes } \\
\text { mellitus }\end{array}$ & 22 & 38 & 34 & 42 & 28 & 49 & 43 & 52 & 38 & 77 & 61 & 82 & 19 & & 34 & \\
\hline Hypertension & 58 & 72 & 78 & 82 & 58 & 72 & 76 & 69 & 45 & 72 & 79 & 82 & 48 & & 66 & \\
\hline Dyslipidemia & 46 & 58 & 52 & 65 & 46 & 57 & 50 & 53 & 36 & 55 & 53 & 67 & 43 & & 47 & \\
\hline Renal failure & N/A & N/A & N/A & N/A & N/A & N/A & N/A & N/A & 3 & 20 & 10 & 30 & 3 & & 12 & \\
\hline Mortality & 4.5 & 7.2 & 8.9 & 9.2 & 4.8 & 9.1 & 9.2 & 16 & 4 & 12 & 7 & 15 & 5.7 & 9.8 & 14 & 13 \\
\hline
\end{tabular}

A: acute coronary syndrome (ACS) alone; B: ACS plus peripheral arterial disease (PAD); C: ACS plus cerebrovascular disease (CVD); D: ACS plus PAD plus CVD; PolyVD: polyvascular disease, all categorical variables represents in percentage (\%); DM: diabetes mellitus; N/A: data not available; ${ }^{*}$ prior/current smoking. Data were collected from $[6,7,9]$.

clinical variables used are incompletely sensitive to identify PAD, approximately half of subjects with $\mathrm{ABI}<0.90$ are asymptomatic, and of those that are symptomatic, only a minority have classic intermittent claudication. The presence of PolyVD is underdiagnosed in our daily practice that may underestimate its true prevalence and impact on the outcome.

\section{Conclusion}

Although PolyVD's patients are high-risk population in the setting of ACS, they received less aggressive therapy. Apart from major bleedings, PolyVD is an independent predictor for adverse hospital outcomes and short- and longterm mortality. Great efforts should be directed to primary and secondary prevention. Looking for the other affected vascular bed in ACS patients will add an important step in risk stratification and management.

\section{Conflict of Interests}

The authors have no conflict of interests.

\section{Funding}

Gulf RACE is a Gulf Heart Association (GHA) project and was financially supported by the GHA, Sanofi Aventis, and the College of Medicine Research Center at King Khalid University Hospital, King Saud University, Riyadh, Saudi Arabia. The sponsors had no role in study design, data collection, data analysis, writing of the report, or submission of the paper. Ethical approvals were obtained prior to the study.

\section{Acknowledgments}

The authors thank the staff in all the participating centers for their invaluable cooperation. Special thanks to Zenaida Ramoso and Kazi Nur Asfina for data coordination and secretarial assistance, and also to the physicians who participated in this registry.

\section{References}

[1] A. El-Menyar, H. Amin, I. Rashdan et al., "Ankle-brachial index and extent of atherosclerosis in patients from the middle east (the AGATHA-ME study): a cross-sectional multicenter study," Angiology, vol. 60, no. 3, pp. 329-334, 2009.

[2] F. G. R. Fowkes, L. P. Low, S. Tuta, and J. Kozak, "Anklebrachial index and extent of atherothrombosis in 8891 patients with or at risk of vascular disease: results of the international AGATHA study," European Heart Journal, vol. 27, no. 15, pp. 1861-1867, 2006.

[3] G. Steg, D. L. Bhatt, P. W.F. Wilson et al., "One-year cardiovascular event rates in outpatients with atherothrombosis," Journal of the American Medical Association, vol. 297, no. 11, pp. 1197-1206, 2007.

[4] P. P. Cacoub, U. Zeymer, T. Limbourg et al., "Effects of adherence to guidelines for the control of major cardiovascular risk factors on outcomes in the REduction of Atherothrombosis for Continued Health (REACH) Registry Europe," Heart, vol. 97, no. 8, pp. 660-667, 2011.

[5] D. L. Bhatt, E. D. Peterson, R. A. Harrington et al., "Prior polyvascular disease: risk factor for adverse ischaemic outcomes in acute coronary syndromes," European Heart Journal, vol. 30, no. 10, pp. 1195-1202, 2009.

[6] A. Meizels, D. M. Zeitoun, V. Bataille et al., "Impact of polyvascular disease on baseline characteristics, management and mortality in acute myocardial infarction. The Alliance project," Archives of Cardiovascular Diseases, vol. 103, no. 4, pp. 207-214, 2010.

[7] D. Mukherjee, K. A. Eagle, E. Kline-Rogers et al., "Impact of prior peripheral arterial disease and stroke on outcomes of acute coronary syndromes and effect of evidence-based therapies (from the Global Registry of Acute Coronary Events)," American Journal of Cardiology, vol. 100, no. 1, pp. $1-6,2007$.

[8] G. Cotter, C. P. Cannon, C. H. McCabe et al., "Prior peripheral arterial disease and cerebrovascular disease are independent predictors of adverse outcome in patients with acute coronary 
syndromes: are we doing enough? Results from the Orbofiban in Patients with Unstable Coronary Syndromes-Thrombolysis in Myocardial Infarction (OPUS-TIMI) 16 study," American Heart Journal, vol. 145, no. 4, pp. 622-627, 2003.

[9] I. Ferreira-González, G. P. Miralda, M. Heras et al., "Investigadores del Estudio MASCARA. Prognosis and management of patients with acute coronary syndrome and polyvascular disease," Revista Espanola de Cardiologia, vol. 62, no. 9, pp. 1012-1021, 2009.

[10] K. F. AlHabib, A. Hersi, A. A. Alsheikh-Ali et al., "Prevalence, predictors, and outcomes of conservative medical management in non-ST-segment elevation acute coronary syndromes in Gulf RACE-2," Angiology. In press.

[11] A. El-Menyar, M. Zubaid, K. Sulaiman et al., "In-hospital major clinical outcomes in patients with chronic renal insufficiency presenting with acute coronary syndrome: data from a registry of 8176 patients," Mayo Clinic Proceedings, vol. 85, no. 4, pp. 332-340, 2010.

[12] C. B. Granger, R. J. Goldberg, O. Dabbous et al., "Predictors of hospital mortality in the Global Registry of Acute Coronary Events," Archives of Internal Medicine, vol. 163, no. 19, pp. 2345-2353, 2003.

[13] S. Uchiyama, S. Goto, M. Matsumoto et al., "Cardiovascular event rates in patients with cerebrovascular disease and atherothrombosis at other vascular locations: results from 1year outcomes in the Japanese REACH Registry," Journal of the Neurological Sciences, vol. 287, no. 1-2, pp. 45-51, 2009.

[14] A. El-Menyar, M. Zubaid, W. Rashed et al., "Comparison of men and women with acute coronary syndrome in six Middle Eastern countries," American Journal of Cardiology, vol. 104, no. 8, pp. 1018-1022, 2009. 


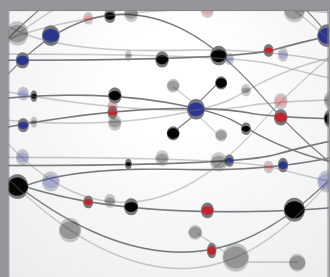

The Scientific World Journal
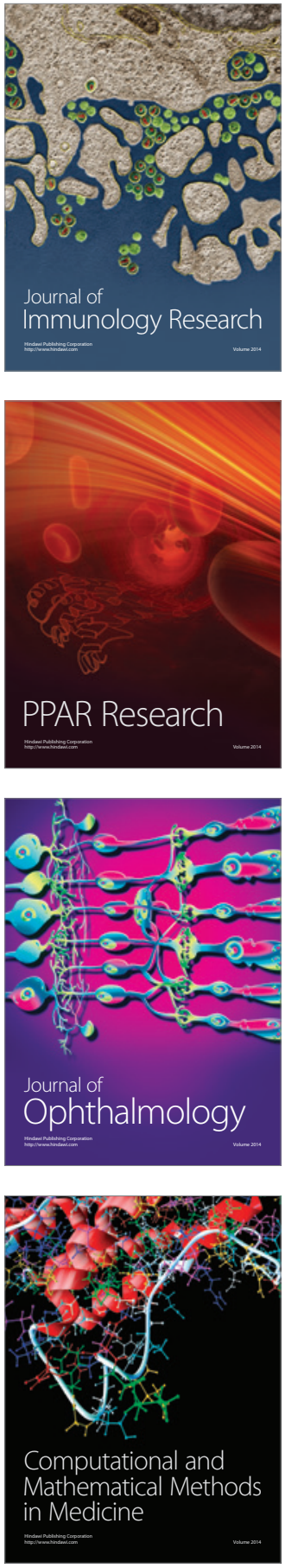

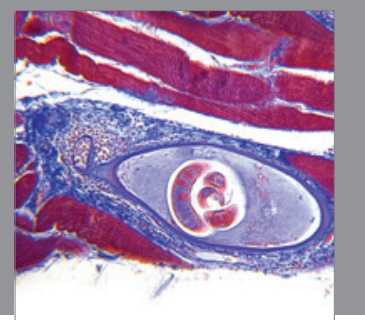

Gastroenterology

Research and Practice
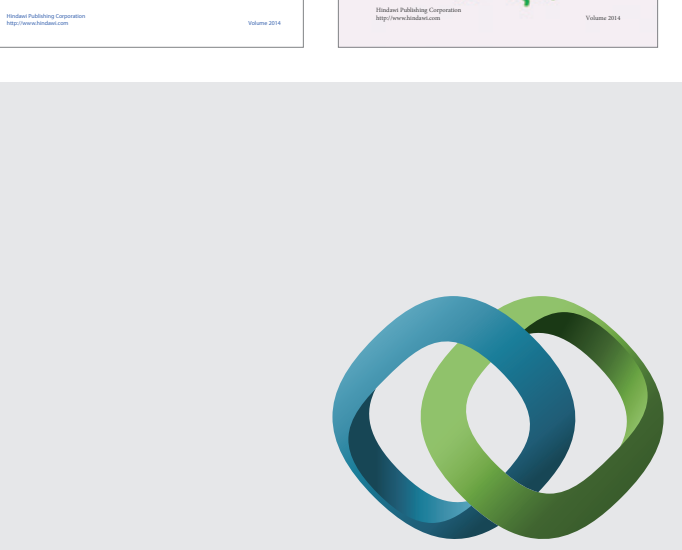

\section{Hindawi}

Submit your manuscripts at

http://www.hindawi.com
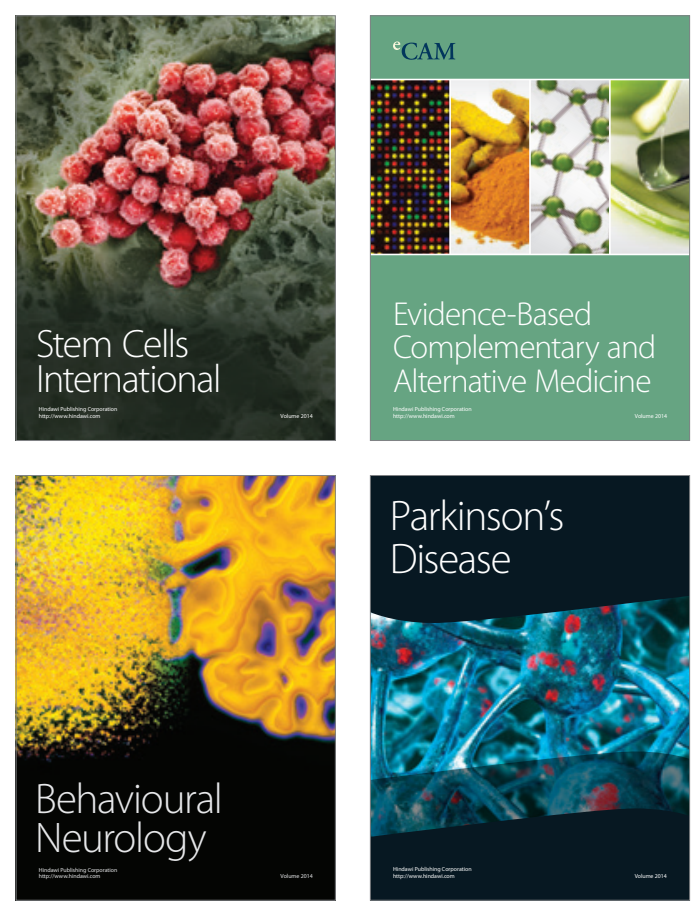

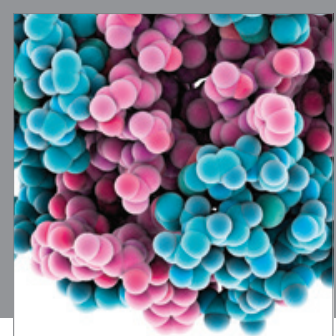

Journal of
Diabetes Research

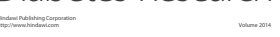

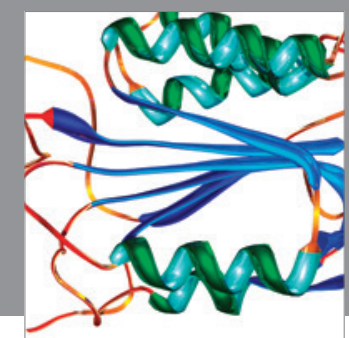

Disease Markers
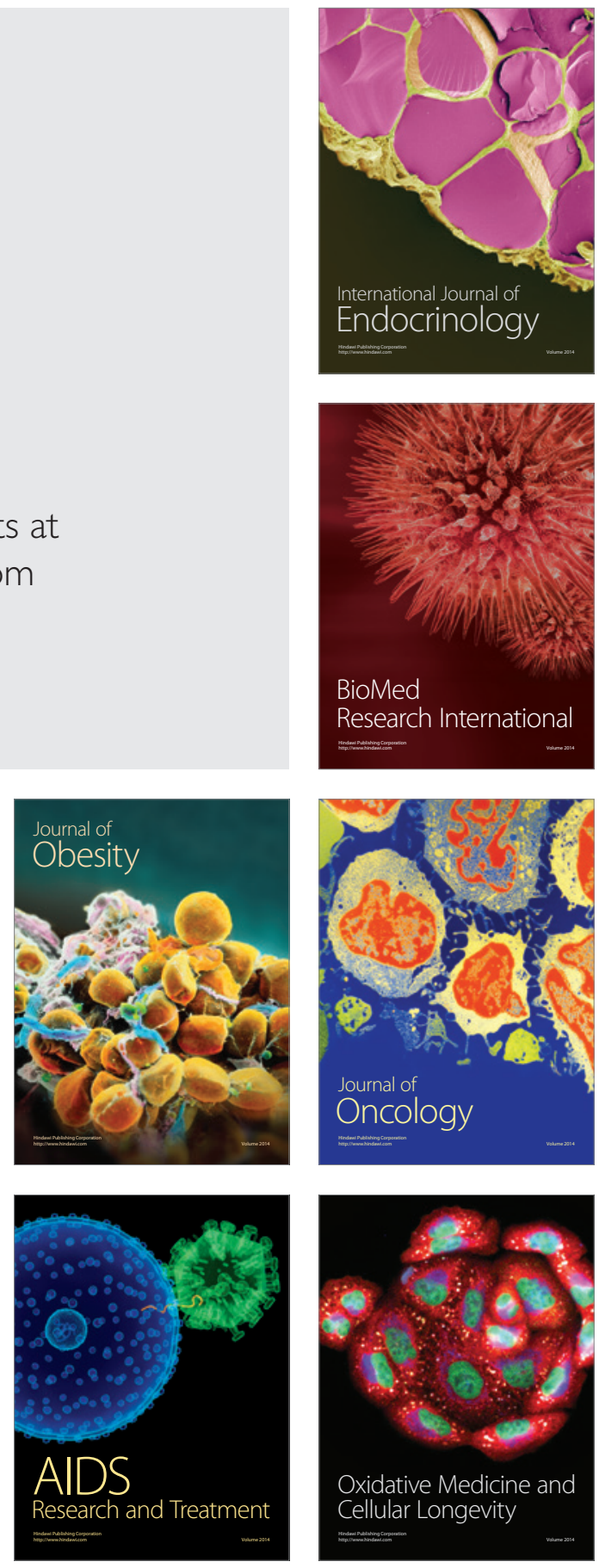